These are level with the surface or very slightly elevated, velvety to the touch and bright red, and are described as "resembling grains of Cayenne pepper." They occur in round, oval or linear groups which progress in serpiginous manner by the formation of new lesions, "satellites," on or just beyond their borders, and by coalescing form gyrate figures. Mixed with the punctate lesions are many linear telangiectases, and the skin between the lesions is often covered by a diffuse pink flush. The bright red lesions fade on pressure; but the deeper ones, darker in color, do not. In some cases very thin atrophic scars have been described, the results of the spontaneous regression of the lesions.

Occurring at any age from infancy to the sixtieth year, the disease may spread slowly or rapidly, or its progress may vary from one to the other extreme. Sensory symptoms are of no importance, never exceeding slight itching or hypersensitiveness of the affected skin. Chilblains of the extremities have been noted in several cases.

Twenty of the twenty-seven cases (incliding Pernet's and mine) were in females, a percentage of 80 , as in two of the cases the sex was not mentioned.

By far the largest number of cases have begun on the extremities, and many have remained imited to a single extremity. Only four cases have begun on the iace, two on the neck and two on the trunk. All parts of the body have been affected except the scalp.

No cause has been discovered. Wise ${ }^{7}$ suggests that disturbances of the internal secretion may be responsible, and that some of the cases may be of toxic origin. The first Anierican case was thoroughly studied and shown to be an angioma-sarcoma, though its subsequent course was wholly benign. Five of the cases were reported as beginning in a "port wine mark."

The diagnosis is easy. No other disease presents such brilliant lesions on a normal or only slightly flushed skin, with groups of lesions spreading at one border and healing behind. Telangiectases due to Roentgen or radium injuries occur on a wrinkled, atrophic skin and lack the punctate lesions, the gyrate figures and the history of spontaneous occurrence and gradual progress.

Telangiectases secondary to other skin diseases, as rosacea, lupus erythematosus or the granulomas, always show enough of the underdying pathologic condition to explain them and never show the punctate lesions, the typical grouping and the serpiginous spreading of angioma serpiginosum. Only two other rare dermatoses show these three characteristics, purpura annularis telangiectodes of Majocchi ${ }^{9}$ and Schamberg's progressive pigmentary dermatosis. ${ }^{10}$ Both of these are distinguished by the presence of hemorrhagic and pigmentary lesions which do not occur in angioma serpiginosum. It is possible that there is a relation between these two diseases and some of the toxic cases of angioma serpiginosum.

Telangiectases from general disturbances, syphilis, ${ }^{1 .}$ lead poisoning, hyperthyroidism, hemophilia, arthritis, cardiovascular disease or diarrhea and edema in children $^{12}$ lack the localization and grouping of angioma serpiginosum. The same may be said of the hereditary

9. Mackee, G. M.: Purpura Annularis Telangiectodes, Jour. Cutan. Dis., 1915, xxxiii, 129.

Dis., 1915, xxxiii, 129.

1913, p. 116.
11. Stokes, J. H.: Generalized Telangiectasia in Association with

11. Stokes, J. H.: Generalized Telangiectas

12. Fearnsides, E. G.: Brit. Jour. Dermat., 1912, xxiv, 35. disease in which cutaneous telangiectasis is associated with lesions of the mucous membranes, hemorrhages and abnormalities of growth. ${ }^{13}$

The prognosis of angioma serpiginosum would seem to be very bad as to cure, judging from the few case reports in which any mention of treatment has been made; but with modern methods so successful in the treatment of vascular nevi, it seems reasonable to hold out a fair prospect of cure. The quartz lamp pressed on the lesion, to produce as deep an effect as possible, deserves the first mention. Radium and the Roentgen ray cautiously applied to the production of a very slight reaction, followed by a long interval of rest from treatment, are also productive of excellent results in nevi. The cautious use of carbon dioxid snow, either alone or in combination with roentgenotherapy, ought to be of value.

The fact that so many of the cases have been reported from England and so few from America means that the disease is diagnosed and reported just in proportion to the degree to which it is brought to the attention of the profession. On the continent no attention has been paid to it; but with the increasing interest in Majocchi's disease it is hardly probable that angioma serpiginosum can be long overlooked.

30 North Michigan Avenue.

\section{THE QUANTITATIVE EFFECT OF SAL- VARSAN ON THE WASSERMANN REACTION OF THE BLOOD *}

$$
\text { JOHN T. KING, JR., M.D. }
$$

It has become generally accepted that in certain cases of syphilis an intravenous injection of salvarsan may produce a positive Wassermann reaction where it has previously been negative. This phenomenon has been called the "provocative" Wassermann test. So widely has this idea been assumed, that one finds conscientious syphilologists who refuse to regard a patient as free from the disease when the routine Wassermann test becomes negative, but insist on a provocative test with negative result at a later date. In some of our large hospitals, patients who show clinical signs of syphilis, but have a negative reaction, are given a dose of salvarsan in an attempt to provoke a positive Wassermann test. An examination of the literature, however, shows that there is a striking lack of exact information on the subject and that very few cases have been reported.

Gennerich $^{1}$ first called attention to the circumstance that Wassermann reactions may be positive following an injection of salvarsan, which previotsly had been negative. He reports twelve cases. In nearly all, only one negative test was done previous to the giving of salvarsan, and the appearance of the "provocative" positive reaction varied from twenty-four hours to fifteen days after the injection, most being observed within the first three days. In five cases, the provoked test is unequivocally called "-, ," in the other seven it is "+ weak," or "+ very weak."

13. Osler: On Telangiectasis Circumscripta Universalis, Johns Hopkirs Hosp. Bull., 1907, xviii, 401.

* From the Medical Clinic of the Johns Hopkins Hospital.

* On account of lack of space this article is abbreviated by omission of a table. The complete article appears in the author's reprints. 1. Gennerich: Berl. klin. Welnnschr, 1910, xlvii, 1735. 
Pease $^{2}$ has collected reports of twenty-four cases from many sources. Of this number, eight were in the primary stage, with initial lesions from three to sixteen weeks previously. Half of these eight cases showed a positive test after a previous negative test; the other half showed a positive, after a weak positive test. In eleven other cases a negative test became positive; in five, a. weak positive became positive. This makes a total of fifteen cases in which a negative test became positive following salvarsan.

The opinions of men with large clinical and laboratory experience regarding the provocative test are varied. Some report cases which they consider authentic instances of provoked tests; others, with equally large experience, are not certain that they have ever encountered a case. In any event, the impression is given that the test is not on an established basis.

Whether there have been few or many cases of provoked Wassermann tests, there are certain valid objections to accepting any of them as proved, for all observations on the effect of antisyphilitic treatment on the Wassermann reaction have been based on tests done at different times. Boas ${ }^{3}$ has shown that the same blood serum, examined with the same reagents and the same technic, at different times gave different results. He proved his point in the following manner: First, he took ten cases with positive Wassermann reactions and did ten successive tests on the same serums with the same reagents. The average variation in the percentage of fixation in the different tests was 22 per cent., the maximum variation in testing a single serum ten times being 100 per cent. In other words, a borderline case, for instance, one having a theoretical complement-fixing power of 50 per cent., might vary between a 100 per cent. positive test and a negative test. Then he tested serums giving a positive reaction with seven different amboceptors in the same test; the average limit of variation was about the same as that above, and the extreme variation was less. Similarly, he tested serums against ten different antigens in oue series, against corpuscles from four different sheep in another, and against eight different complements in another. He concludes that the variation resulting from using different amboceptors and different antigens in the same test was no greater than that resulting from repetition of a test with the same reagents, and that the variation from using corpuscles of different sheep and different complements was even less.

Craig ${ }^{4}$ titrated the strength of the Wassermann reaction in the blood of untreated syphilitics, and obtained wide variations in his results in the scope of two weeks, although he used the same reagents. Craig attributed the fluctuations in complement fixation to changes in the actual amount of complement-binding substance in the blood. But here again the question arises whether or not such variations may be due to slight variations in the delicately balanced test from time to time. Such, indeed, seems to be the case, for work by Haller," which appeared while this paper was being written, furnishes good evidence that the factor in the blood which produces a positive Wassermann test is a practically constant element. This author collected specimens of blood on seven successive days

2. Pease, M. C., Jr.: Med. Rec., New York, 1914, 1xxxv, 982.

3. Boas, Harald: Die Wassermannsche Reaktion, 1913.

4. Craig, C. F.: Variations in the Strength of the Wassermann Reaction in Untreated Syphilitic Infections, TIIE. Journal A. M. A., April 18, 1914, p. 1232 .

5. Haller, D. A.: Variations in the Strength of Positive Wassermann Reactions in Cases of Untreated Syphilis, The Journal A. M. A., March 18, 1916, p. 882 . from patients with known positive Wassermann tests, the patients receiving no treatment. The serums were then all tested with the same hemolytic system. He showed that the variation from day to day in the strength of the Wassermann reaction in a given individual is inappreciable; that the variations from month to month are slight, and that variations encountered in daily titration of the fixing unit are due to variation in the hemolytic system, provided the antigen is the same. Such results show that the fluctuations observed by Craig were probably due to variations in the test itself from day to day, and not to any fluctuation of the complement-binding substance in the blood.

To avoid this error, all the results reported here were obtained from titrations done at the same time, and under identical conditions. Since one cannot calculate the titer of a serum which gives a negative test, it was decided to select patients with known positive Wassermann reactions and to determine the strength of the fixing power before and at various times after the giving of salvarsan.

\section{METHOD}

The series includes patients with each of the ordinary syphilitic manifestations - hereditary, primary, secondary and tertiary syphilis, and also with syphilis of the central nervous system and of the aorta, tabes dorsalis and paresis. In some, the test was done before the administration of salvarsan, four, eighteen, twenty-four and forty-eight hours after the injection. The time interval varied in all cases, to allow for the patient's rest. Most of the cases, however, were followed over five days, specimens being taken before treatment, four, twenty-four, forty-eight hours and five days later. Some cases were followed for several weeks.

In most cases, the blood specimens were obtained in a capillary tube. After it had been cleaned with alcohol and dried, the lobe of the ear was punctured with a stilet. The filled tubes were sealed promptly at both ends. A few specimens were obtained by venipuncture, and kept in a closed sterile test tube. All bloods were put inmediately into the same receiver in the same ice box. After a full series of specimens from any case was obtained, they were all tested at the same time. In some instances blood was preserved several weeks on ice and titrated in the same tests with specimens obtained after weeks of treatment.

The test was made as follows: The patient's serum was diluted with 4 parts of 0.85 per cent. sterile salt solution, and inactivated in a water bath at $56 \mathrm{C}$. for twenty minutes. The serum was then distributed as follows: Into the first tube was put 0.25 c.c. of the serum of the first dilution, having a concentration of $1: 5$. Successive tubes contained 0.25 c.c. of dilited serum in concentration of $1: 10,1: 20,1: 40,1: 80$ and $1: 160$, and, in some cases, $1: 320$ and $1: 640$. The actual amount of serum in the tubes, therefore, was 0.05 c.c., 0.025 c.c., 0.0125 c.c., 0.00625 c.c., 0.003125 c.c., 0.0015625 c.c., and sometimes less. Alcoholic extract of beef heart was used as antigen in most of the tests; this contained 0.2 per cent. cholesterin; in a few tests at first, extract with 0.4 per cent. cholesterin was used. Guinea-pig complement and the rabbit-sheep hemolytic system were employed, 0.25 c.c. quantities of each reagent being used. The usual controls were carried out. Beth the preliminary and final incubation periods were one-half hour each. The results were read after sedimentation was complete, usually after one to two 
hours. The percentage of fixation in each tube was read as nearly as possible, 100 per cent. representing an absolutely nonhemolyzed tube. Therefore, by reading down the column of figures under any given date, one finds the percentage of complement fixation in tubes containing diminishing quantities of serum.

\section{COMMENT}

Titrations were carried out in sixteen different cases of syphilis, and before and after twenty administrations of salvarsan. In some cases the observations were made over a period of two days, in more cases for five days after administration. There was one case each of primary and secondary syphilis, four of gummas, one of tabes dorsalis, one of general paresis, two of hereditary syphilis, two of vascular and two of meningeal syphilis, including one of syphilis of the central nervous system, and two cases diagnosed only by the Wassermann reaction. Thirteen doses of salvarsan were followed by no more fluctuation of the titer than is explainable by the inherent limit of error of the test; in five, the end titration showed a weakening; in one case there was an increase in titer, followed by a return to the original strength. One case is included in which the end point was not reached. The effect of treatment on the strength of the Wassermann reaction in these cases was strikingly slight, except in the case of primary syphilis. Although this patient had already received two injections of salvarsan, his blood still showed a strong reaction, which became much weaker after this treatment. In this case, the initial lesion had completely healed before the titrations were begun, and there were no secondary manifestations; yet, though the Wassermann titer was stronger than in many of the cases in other stages, it became more rapidly weaker than it did in any other case.

In Cases 1 and 15 (Footnote 15), fluctuations in titer were noted, not in any one test, but in different series. In Case 1, the titer was constant for five days, the specimens being titrated in the same test. Two days later, the collection of a second group of specimens was begun and carried out for five days; this group differed from the first group, but the titer within the group was constant; a third group, begun two days later, showed constant titer in itself, but differed from the second group by being like the first.

In Case 15, the first group of specimens showed constant titer; a second group begun three days later gave no fixation throughout; one blood specimen of the second group, however, titrated with blood collected thirteen days later, gave the same amount of fixation as the last specimen ( 25 per cent.), in spite of intervening therapy. Therefore, it seems that fuctuations in titer do occur when titrations are done at different times, in spite of the fact that there are no essential fluctuations in the actual complement-fixing power of the blood. Such must be interpreted as being due mainly to changes in the delicacy of the test rather than to fluctuations in the condition of the blood. Most of the cases here presented show practically no change in the strength of the Wassermann reaction from day to day, in spite of treatment. This supports the work of Haller referred to above.

In only one case in this series was there any increase in titer of the reaction following an injection of salvarsan, although many of the cases were of the types commonly said to develop a provocative test. In this one test, the result is not easy to interpret, as the rise in titer came one hour after salvarsan, the titer being the same the next day and weaker on the second day, which is said to be the optimum time for demonstration of the provocative reaction. Nor can we assume that, had this patient previously had a negative Wassermann reaction, this would be a case of true provocation, for the increase in titer was so slight that the resulting point might still have been below the threshold of positivity.

In view of such results, nothing is found definitely to support the assumption that provocative Wassermann reactions do occur; yet it would be presumptuous to assert that an increase in titer may not occasionally occur. The series does not contain a case of so-called Herxheimer reaction, and it is conceivable that such a phenomenon might be associated with a provocative test. The results do suggest, however, that the complement-binding substance in syphilitic serum is a relatively constant factor-at least not readily increased by salvarsan injections. Therefore, it is probable that most, if not all, of the salvarsan given in the hope of calling forth a positive after a negative Wassermann test is wasted.

How, then, can the results reported in the literature of the provocative reaction and its common acceptance be accounted for? When one examines the reports of Gennerich, it is found that the reactions which were compared were done at different times. This brings in the error which Boas found resulted from pure repetition of the test, as well as the fluctuation which Craig found from day to day, when he titrated serums at different times. The fluctuations which Gennerich observed after salvarsan are not more striking than those observed by Boas and by Craig in untreated cases. Therefore, it seems that, to prove that the provocative Wassermann reaction is a fact, it is imperative that the blood specimens before and after salvarsan be examined in the same test.

\section{CONCLUSIONS}

1. In most cases little change occurs in the strength of the Wassermann reaction during the first five days following the administration of salvarsan. In this series of twenty treatments, only one case, in the primary stage, showed a marked weakening of the test.

2. Some previously untreated cases may be given prolonged salvarsan therapy with very little weakening of the Wassermann reaction. Such cases may, however, show striking improvement symptomatically.

3. In this series, only one insignificant temporary increase (provocative reaction) in the complementbinding substance could be demonstrated, following the administration of salvarsan.

4. It is improbable that, over short periods of time, there occurs any marked spontaneous fluctuation in the amount of complement-fixing substance in the blood of syphilitics.

5. Definite proof of the existence of the provocative Wassermann reaction following salvarsan is not at hand at the present time.

As Barnum Said.- "When 'Beauty Specialist,' in one of those faked 'Health Hints' or 'Beauty Hints,' and other 'Madames' or 'Mademoiselles,' whose contributions are generally signed 'Adv.,' suggest to 'Marie' or 'Discouraged' that a little 'kardene,' 'parnotis,' or 'almazoin,' will reduce those enlarged pores, some one is about to be taken in, the stuff with the funny name being the joker in the disguised advertisement."-William Brady. 